\title{
sciendo
}

\section{Clinical manifestations of neurofibromatosis type 1 - a diagnostic and management challenge}

\begin{abstract}
Introduction. Neurofibromatosis type 1 (NF1), caused by mutation of the tumour-suppressor gene encoding neurofibromin, is an autosomal dominant disorder affecting various organs.

Aim. The aim of the study was to discuss the clinical symptoms of NF1 based on seven cases of the disease with regard to the literature.

Material and methods. We analyzed retrospectively patients with NF1 (4 females and 3 males) aged 19-52 who were treated at Endocrinology Department between 2003 and 2017. The diagnosis was made in childhood (4 patients) or in adolescence (3 cases), based on clinical symptoms and genetic tests.

Results. Five patients had a positive family history of NF1, two cases represented spontaneous mutation. All of analyzed subjects presented café-au-lait spots and neurofibromas on the body. We observed neurological disorders such as: epilepsy (2 patients), Arnold-Chiari malformation (1 man), benign brain neoplasms ( 2 persons). Optic gliomas appeared in two cases. Tumours were also found in other organs, including the uterus ( 2 women), the lung, the adrenals, the pituitary and the parathyroid gland (with signs of primary hyperparathyroidism) - each tumour in another patient. Four subjects suffered from cognitive impairment. Skeletal manifestations of neurofibromatosis type 1 such as scoliosis (1 man) and short stature (6 patients) have been noted as well. Five patients presented thyroid disorders - hypothyroidism due to Hashimoto's disease (4 patients), toxic nodular goiter (1 woman). Other clinical symptoms e.g. vitiligo, alopecia areata and coarctation of aorta have also been found.

Conclusions. Variety of clinical symptoms causes that NF1 still remains a diagnostic and management challenge for many physicians. Therefore, multidisciplinary approach is needed to optimize patients' treatment.
\end{abstract}

Keywords: autoimmune disorders, epilepsy, neurofibromatosis, thyroid, tumours.

DOI: $10.2478 /$ pjph-2018-0020

\section{INTRODUCTION}

Neurofibromatosis type 1 (NF1; von Recklinghausen's disease) is an autosomal dominant disorder with a penetrance of almost $100 \%$ and without sex or racial predilection $[1,2]$. It was first described by pathologist - Friederich Daniel Von Recklinghausen in 1882 [1]. The prevalence of the disease is estimated to be 1 in $2500-3500$ births $[1,3]$. NF1 is caused by dominant loss-of-function mutations of the tumour-suppressor gene NF1 located at the 17q11.2 chromosome [1,4] It is well known that NF1 gene encodes neurofibromin, a protein expressed in neurons, Schwann cells, oligodendrocytes and leukocytes, which takes part in intracellular processes such as: RAS-cyclic AMP pathway, adenyl cyclase, ERK/MAP kinase cascade and the cytoskeletal assembly [5]. However, only 50\% of the NF1 patients has a positive family history of the disease, the rest of cases represents spontaneous mutations [2,3]. NF1 affects various organs such as: skin, nervous system, vision organ, skeletal and vascular system as well [1-4]. Patients are at an increased risk of developing both benign and malignant tumours, therefore NF1 is also termed as tumour predisposition syndrome [4]. The clinical manifestations of the disease differ significantly from mild lesions to several complications and function impairment, even in members of one family $[1,4]$.

\section{AIM}

The aim of the study was to discuss the clinical symptoms and signs of neurofibromatosis type 1 based on seven cases of the disease with regard to the literature.

\section{MATERIAL AND METHODS}

We analyzed retrospectively seven cases of patients with NF1 (4 females and 3 males) aged 19-52 who were treated at the Endocrinology Department between 2003 and 2017. In studied subjects, the diagnosis was made based on clinical symptoms and confirmed by genetic tests. Four patients ( 3 women and 1 man) were diagnosed in childhood. In three cases ( 1 woman and 2 men) the diagnosis was made in adolescence. Two participants (1 woman and 1 man) belonged to one family.

${ }^{1}$ Chair and Department of Endocrinology, II Faculty of Medicine with English Language Division, Medical University of Lublin, Poland

${ }^{2}$ Department of Internal Medicine in Nursing, Faculty of Health Sciences, Medical University of Lublin, Poland 


\section{RESULTS}

In analyzed group of patients, five subjects had a positive family history of NF1, two cases represented spontaneous mutations. All of them suffered from neurocutaneous manifestations of the disease such as café-au-lait spots and soft tissue cutaneous nodules on the body (neurofibromas).

The neurological disorders have been also observed. Two patients demonstrated epilepsy but they did not require any treatment at that time. In one man, Arnold-Chiari malformation has been diagnosed. Benign brain neoplasms developed in two persons.

Moreover, optic gliomas appeared in two cases. One man had a surgery and received radiotherapy due to optic chiasma astrocytoma. Left eye blindness, as a complication of the management, has been occurred. One woman was operated because of bilateral optic gliomas, without complications.

Except for brain tumours and optic gliomas, many neoplasms developed in other organs. One subject is observed because of nodule $(19 \times 11 \mathrm{~mm})$ which is localized in the apex of left lung. Two women had leiomyomas in the uterus. In one man right adrenalectomy has been performed due to lesion of the adrenal gland $(55 \times 39 \times 48 \mathrm{~mm})$. There are no symptoms of adrenal insufficiency at present. The diagnosis of pheochromocytoma has been made just after surgery based on histopathological examination. No episodes of the increase in blood pressure or permanent hypertension in the past medical history have been observed before adrenalectomy. Furthermore, other endocrine glands have been involved in the disease. Non-functioning pituitary adenoma has developed in one woman. Another patient has been operated because of primary hyperparathyroidism due to parathyroid adenoma $(18 \times 23 \times 39 \mathrm{~mm})$.

Four patients manifested cognitive impairment. Moreover, we observed the skeletal manifestations of NF1 such as scoliosis - in one subject and short stature - in six cases. Most of analyzed patients suffered from thyroid disorders: four of them had hypothyroidism due to Hashimoto's disease, one woman had toxic nodular goiter. Other autoimmunological disorders, including vitiligo - in two cases and alopecia areata - in one woman, have been found as well. We have also noted the vascular manifestations of NF1. One man has been operated after birth due to coarctacion of the abdominal aorta.

\section{DISCUSSION}

The diagnosis of neurofibromatosis type 1 can be made based on genetic testing or clinical symptoms [3]. The variety of symptoms may be influenced by environmental factors, modifier genes or both [4]. Many disease features are more prevalent in older patients $[4,6]$. What is more, de novo NF1 cases usually lead to milder or atypical NF1 manifestations [7].

In 1987, seven cardinal diagnostic criteria for NF1 were established and later in 1997, they were updated by the National Institutes of Health Consensus Development Conference [3,8]. The criteria have been shown in Figure 1.

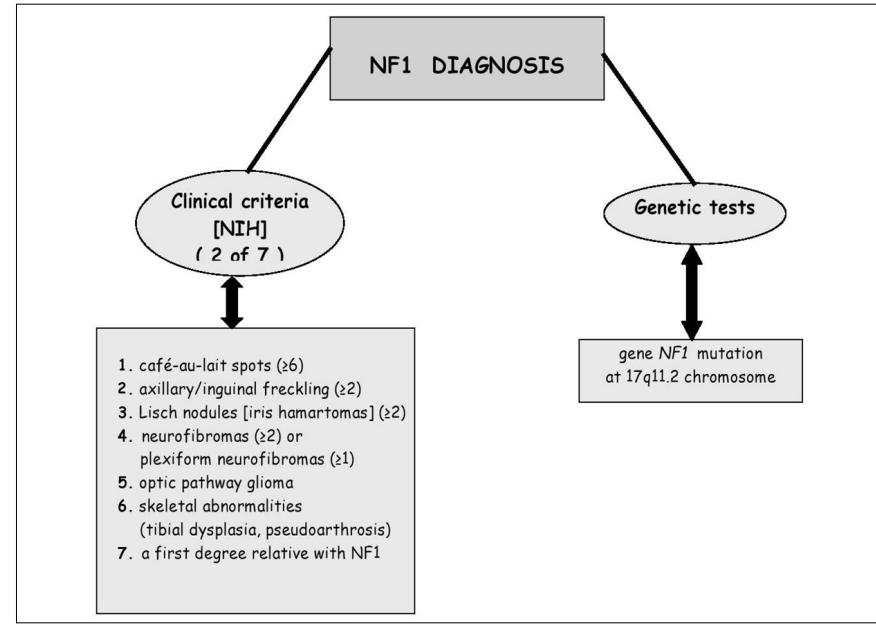

FIGURE 1. Diagnostic criteria for neurofibromatosis type 1.

At least two of them must be present to give the diagnosis of NF1 [6]. All of our subjects have been diagnosed based on genetic and clinical findings either in childhood or in adolescence.

Typical for NF1 pigmented lesions such as café-au-lait spots which borders may be irregular with colour range from light to dark brown and freckles (Crowe's sign), usually appear during the first years of life or are present at birth [1]. Their prevalence reaches $99 \%$ and $85 \%$, respectively [3]. In the studied group café-au-lait spots were common findings because all of patients had these birthmarks from early age.

Neurofibromas were also present in all described subjects. These benign tumours are composed of Schwann cells, perineurium-like cells, fibroblasts and mast cells as well [9]. In NF1 two types of neurofibromas may develop: 'discrete' named also 'localized' neurofibroma and 'plexiform' neurofibroma [1,3]. A 'localized' lesions, presented as a focal mass with margins, appear usually in late childhood or in adolescence, mostly on the skin. Cutaneous ones greatly affect quality of life [9] and this type was present in our patients. 'Plexiform' neurofibromas are locally invasive tumours which spread along the peripheral nerve and may affect its structure. The risk of malignization is estimated to be $2-5 \%$ [1]. No one of mentioned participants had them.

Endocrine disorders are the main cause that patients with neurofibromatosis type 1 attend to endocrinologists. The data suggest that $20-25 \%$ of cases of the sporadic pheochromocytoma is connected with somatic NF1 mutations [10]. It is also well known that from $0.1 \%$ to $5.7 \%$ of patients with NF1 develop pheochromocytoma. The prevalence is higher in those with hypertension and reaches then to $20-50 \%$ [11]. Only one patient in the studied group had this neoplasm. What is more, the diagnosis was made after the surgery because there were no episodes of hypertension in the past medical history [11].

The most common endocrine pathology in our subjects was hypothyroidism due to Hashimoto's disease which occurred in $57 \%$ of analyzed group of patients. Generally, autoimmune diseases are rare in neurofibromatosis type 1. Only few cases with the coexistence of NF1 and Hashimoto's thyroiditis have been described so far [6]. Besides chronic thyroiditis, systemic lupus erythematosus, multiple sclerosis, membranous glomerulonephritis, IgA nephropathy, mixed connective tissue disease, juvenile arthritis, autoimmune hemolytic anemia, bullous pemphigoid and vitiligo have been reported in subjects 
with NF1 [4]. The last one appeared in two studied participants. Moreover, one patient had alopecia areata. The pathophysiology of autoimmune disorders in NF1 is not clear but it is known that abnormal production of neurofibromin leads to suppress expression of Fas-ligand, which prevents apoptosis of CD4+ T-cells. Decreased T-cells apoptosis due to abnormal neurofibromin production may be an underlying factor for the development of the autoimmune diseases [6].

A combination of primary hyperparathyroidism and NF1 is also a rare phenomenon [12]. In our study, in one woman with laboratory signs of primary hyperparathyroidism including: elevated level of PTH $(454 \mathrm{pg} / \mathrm{ml} ;$ n. 14-72), hypercalcaemia (Ca-12.20mg/dl; n.8.6-10.4), hypophosphatemia (P-1.30mg/dl; n.2.4-5.1), hypercalciuria (urinary calcium-608.0mg/24-hours; n.100-300), adenoma $(18 \times 23 \times 39 \mathrm{~mm})$ of the right parathyroid gland has been diagnosed. The patient has been operated, unfortunately the surgery was not radical and reoperation is actually planned.

Various lesions in the thyroid gland such as papillary carcinoma, follicular carcinoma and neurofibromas of the thyroid gland, in association to NF1 have been also described [13]. In the studied group, in one woman the surgery has been performed due to retrosternal toxic nodular goiter which caused the impression of the trachea.

Moreover, the analyzed group of patients other neoplasms have been observed. In two women - leiomyomas in the uterus and in one man - nodule of the lung have been appeared. In another two patients benign brain tumours have been found as well.

Our findings are consistent with previous reports. For instance, the public mutation database COSMIC has revealed that: $5.25 \%$ of subjects with NF1 had CNS tumours, 5.04\% - endometrium neoplasms, $8.0 \%$ - lung tumours and $9.09 \%$ - thyroid lesions [11]. Malignant peripheral nerve sheath tumours (2-5\%) and malignant gliomas (1-2\%) may be also present [3]. It has been proved that subjects with NF1 are at an increased risk of developing both benign and malignant tumours because a loss of neurofibromin results in increased mitogenic signaling and leads to increased cellular proliferation or differentiation [14].

Optic nerve gliomas are one of the diagnostic criterion of NF1 [6]. They may be localized in prechiasmal, chiasmal and postchiasmal regions. Optic pathway glioma occurs in $15 \%$ of cases [3]. In the studied group of patients: one man had a surgery and received radiotherapy due to optic chiasma astrocytoma, one woman suffered from bilateral optic gliomas. Tumours in this localization may result in blindness [1] and this complication we observed in one case.

It is suggested that NF1 is a risk factor for epilepsy. Some authors found that seizures occur in $4-7 \%$ of cases with neurofibromatosis type 1 and the prevalence is higher than in general population (1-2\%). However, they are commonly relatively easy to treat with antiepileptic drugs [8]. Our results are in accordance with these statements because epilepsy has been diagnosed in two cases but none of them require antiepileptic therapy at present.

Except for mentioned above neurological disorders, we observed Arnold-Chiari malformation type I (ACM-I) in one patient. This pathology is characterized by caudal migration of the cerebellar tonsils. Headache, weakness and sensory abnormalities are the most common symptoms. Recent data suggested that ACM-I is not a rare finding in subjects with NF1 and may affect from $2 \%$ to even about $8 \%$ of patients with NF1 [15].

Some patients with NF1 suffer from learning/behavioral problems. Diggs-Andrews and Gutmann [3] find that approximately $70 \%$ of children with NF1 have cognitive deficits compared to $10 \%$ of children in general population. It may be manifested as difficulties with attention, executive function (planning and concept formation), language, visual perception and learning as well. An increased incidence of autism, ADHD (attention-deficit hyperactivity disorder) or impulsive behavior has been also reported [3]. Cognitive impairment has been diagnosed in our patients as well and affected four subjects.

Approximately $50 \%$ of patients with NF1 have skeletal disturbances. Short stature (30\%), macrocephaly - enlarged heads $(45 \%)$ and scoliosis $(5-10 \%)$ are common pathologies [3]. In the analyzed group, the most common disorder was short stature which occurrence reached $86 \%$. Only one patient suffered from scoliosis. Abnormal hypothalamic-pituitary axis function has been hypothesized to play a causative role in the pathogenesis of short stature [16]. Hegedus et al. [16] showed in animal study that mice with loss of neurofibromin expression in hypothalamus were smaller due to reduced growth hormone-releasing hormone, pituitary growth hormone $(\mathrm{GH})$ and liver insulin-like growth factor-1 (IGF1) production. These results are consistent with other study which was conducted in children with NF1, approximately $80 \%$ of these children $(15 / 19$ patients) were found to have growth hormone (GH) deficiency in the absence of suprasellar abnormalities on neuroimaging [16].

NF1 may be also accompanied by vascular abnormalities (0.4-6.4\%) which are usually asymptomatic. It is known that neurofibromin expression is present in the endothelial and smooth muscle cells of blood vessels. The pathophysiology of vascular change in subject with NF1 is characterized by fibromuscular dysplasia and a thickening of the tunica intima of vessel walls [17]. Recent data suggest that the most common pathologies are: abdominal aortic coarctation, aneurysms, stenoses of the aorta, renal arteries and mesenteric vessels, whereas $12 \%$ of vascular disturbances is represented by abdominal aortic coarctation or aneurysms. What is more, aorta pathologies usually occur in younger patients. This observations are similar to our findings because in our study, one man has been operated due to coarctacion of the abdominal aorta after birth. It is suggested that operative treatment of symptomatic patients with vascular lesions or with large aneurysms should be performed to avoid fatal complications [14].

There is no medical treatment for neurofibromatosis type 1. Surgical resection of the lesions is indicated when they cause patient's function deterioration, pain or cosmetic disfigurement $[1,2]$. The special care must be taken of possibility of tumours malignant transformation which is fortunately low (3-5\%) but the risk of malignancies is higher than in general population (these patients require only one mutation to occur cancers because the second is inherited) [2]. Radiotherapy and chemotherapy are not indicated [1].

The survival in patients with neurofibromatosis type 1 is shorter compared with the general population and a median age is estimated to be about 60 years. The most common cause of death is malignancy often from connective and soft-tissue neoplasms. Vascular disease is the second leading cause of death, especially among those individuals aged about 40 years old [14]. 


\section{CONCLUSION}

We analyzed only seven cases of patients with neurofibromatosis type 1 . Despite the fact that this was a small group of subjects, most of our findings were consistent with previous reports. From an endocrine point of view, the most common pathology in the studied group of patients was hypothyroidism due to Hashimoto's disease. Hence, we suggest that in each newly diagnosed subject with NF1, a screening test of this disease (e.g. determination of TSH and anti-thyroperoxidase antibodies levels) should be performed.

We conclude that the variety of clinical symptoms causes that NF1 still remains a diagnostic and management challenge for many physicians. Therefore, multidisciplinary approach is needed to optimize patients' diagnosis and treatment.

\section{REFERENCES}

1. Ghalayani P, Saberi Z, Sardari F. Neurofibromatosis type I (von Recklinghausen's disease): a family case report and literature review. Dent Res J (Isfahan). 2012;9:483-8.

2. Dadlani R, Sadanand V, Ghosal N, Hegde SH. Congenital giant plexiform neurofibroma with occipital calvarial dysplasia in association with meningoencephalocele in neurofibromatosis Type 1 and segmental neurofibromatosis. J Neurosurg Pediatrics. 2013;12:458-64.

3. Diggs-Andrews KA, Gutmann DH. Modeling cognitive dysfunction in neurofibromatosis-1. Trends Neurosci. 2013;36:237-47.

4. Pasmant E, Vidaud M, Vidaud D, Wolkenstein P. Neurofibromatosis type 1: from genotype to phenotype. J Med Genet. 2012;49:483-9.

5. Crona J, Delgado Verdugo A, Maharjan R, et al. Somatic mutations in HRAS in sporadic pheochromocytoma and paraganglioma identified by exome sequencing. J Clin Endocrinol Metab. 2013;98:E1266-71.

6. Nabi J. Neurofibromatosis type 1 associated with Hashimoto's thyroiditis: coincidence or possible link. Case Rep Neurol Med. 2013; 2013:910656. doi: 10.1155/2013/910656. PubMed PMID: 23691379; PubMed Central PMCID: PMC3652086.

7. Messiaen L, Vogt J, Bengesser K, et al. Mosaic type-1 NF1 microdeletions as a cause of both generalized and segmental neurofibromatosis type-1 (NF1). Hum Mutat. 2011;32:213-9.

8. Ostendorf AP, Gutmann DH, Weisenberg JL. Epilepsy in individuals with neurofibromatosis type I. Epilepsia. 2013;54:1810-4.

9. Zehou O, Fabre E, Zelek L, et al. Chemotherapy for the treatment of malignant peripheral nerve sheath tumors in neurofibromatosis 1: a 10-year institutional review. Orphanet J Rare. 2013;8:127.

10. Burnichon N, Buffet A, Parfait B, et al. Somatic NF1 inactivation is a frequent event in sporadic pheochromocytoma. Hum Mol Genet. 2012;21:5397-405.

11. Welander J, Söderkvist P, Gimm O. The NF1 gene: a frequent mutational target in sporadic pheochromocytomas and beyond. Endocr Relat Cancer. 2013;20:C13-7.

12. Altinova AE, Toruner F, Cimen AR, et al. The association of neurofibromatosis, bilateral pheochromocytoma and primary hyperparathyroidism. Exp Clin Endocrinol Diabetes. 2007;115:468-70.

13. Güler S, Yeşil G, Önal H. Endocrinological evaluations of a neurofibromatosis type 1 cohort: Is it necessary to evaluate autoimmune thyroiditis in neurofibromatosis type 1? Balkan Med J. 2017;34:522-6.

14. Oderich GS, Sullivan TM, Bower TC, et al. Vascular abnormalities in patients with neurofibromatosis syndrome type I: clinical spectrum, management, and results. J Vasc Surg. 2007;46:475-84.

15. Miraglia E, Fabbrini G, Di Biasi C, et al. Chiari type 1 malformation in Neurofibromatosis type 1: experience of a center and review of the literature. Clin Ter. 2016;167:e6-10.

16. Hegedus B, Yeh TH, Lee da Y, et al. Neurofibromin regulates somatic growth through the hypothalamic-pituitary axis. Hum Mol Genet. 2008;17:2956-66.

17. Makino K, Kurita N, Kanai M, Kirita M. Spontaneous rupture of a dissecting aneurysm in the superior rectal artery of a patient with neurofibromatosis type 1: a case report. J Med Case Rep. 2013;7:249. doi: 10.1186/17521947-7-249.

\author{
Corresponding author \\ Anna Maria Dąbrowska \\ Chair and Department of Endocrinology, Medical University of Lublin \\ 8 Jaczewskiego St., 20-954 Lublin, Poland \\ tel. +48604676091 \\ E-mail: dabrowska.ania.m@gmail.com
}

